\title{
Harmonics and voltage stability analysis in power systems including thyristor-controlled reactor
}

\author{
M UZUNOGLU \\ Yildiz Technical University, Electrical-Electronics Faculty, Electrical \\ Engineering Department, 34349 Besiktas, Istanbul, Turkey \\ e-mail: uzunoglu@yildiz.edu.tr
}

MS received 28 October 2003; accepted 5 May 2004

\begin{abstract}
In this study, non-sinusoidal quantities and voltage stability, both known as power quality criteria, are examined together in detail. The widespread use of power electronics elements cause the existence of significant non-sinusoidal quantities in the system. These non-sinusoidal quantities can create serious harmonic distortions in transmission and distribution systems. In this paper, harmonic generation of a static VAR compensator with thyristor-controlled reactor and effects of the harmonics on steady-state voltage stability are examined for various operational conditions.
\end{abstract}

Keywords. Harmonics; stability; thyristor-controlled reactor.

\section{Introduction}

It is not only required to meet the demand for electrical energy but also to improve its quality. Today, power quality studies are becoming a growing concern. The present paper focuses on two parameters affecting power quality - harmonics and voltage stability.

Static VAR compensators (SVCs) are applied on transmission systems to improve voltage control and system stability during both normal and contingency system conditions. Most SVCs use a thyristor-controlled reactor configuration to provide continuous control of the reactive power compensation level. The thyristor-controlled reactor (TCR) produces harmonic currents because thyristors only allow conduction in the reactor for a portion of the cycle. Harmonic current magnitudes vary as the firing angle of the thyristors is varied. A comprehensive harmonic study includes evaluation of possible harmonic concerns over the full range of firing angles and possible system conditions. Generally, harmonic filtering is accomplished with capacitors connected in parallel with the TCRs. For transmission applications, the filters might include tuned filters at the fifth and seventh harmonics along with a high pass filter for higher order components.

Use of a static compensator with a thyristor-controlled reactor (TCR) has been known to be an effective and reliable means to ensure power system voltage stability. This device is essentially a variable reactor that can adjust its reactive power consumption. The adjustment is realised through the firing angles of thyristors that normally operate in partial conducting 
states, and thereby produce harmonics (Miller 1982). The generated harmonics depend critically on the control characteristics of a static VAR compensator (SVC) with a fixed-capacitor thyristor-controlled reactor (FC-TCR). However, this makes the firing angles dependent on the network load flow conditions and harmonic voltage distributions (Xu et al 1991). Poor selection of firing angles can lead to increase in the amount of effective harmonic production of the TCR. From the operation point of view of the system, we need to model the harmonics accurately (Uzunoglu et al 1999).

In the literature, work on stability analyses has mostly been done on transients. In this study, however, steady-state stability analysis of a system including the harmonics of FC-TCR has been carried out employing a new algorithm. This paper aims to determine the effects of non-sinusoidal quantities on voltage stability, due to a FC-TCR, by means of a synthesis of harmonic power flow analysis and voltage stability analysis based on the Newton-Raphson method.

\section{Thyristor-controlled reactor as harmonic source}

Thyristor-controlled reactors, which have the ability to ensure continuous and fast reactive power and voltage control, can increase the performance of the system in different ways such as control of transient over-voltages at the power frequency, prevent ion of voltage collapse, increase in transient stability and decrease in system oscillations. Static VAR compensators consisting of thyristor-controlled reactors are used for balancing the three-phase systems.

The basic static VAR system (SVS) consists of a static switch in series with an inductor. This is normally called a phase-controlled reactor or thyristor-controlled reactor (TCR). This basic TCR is illustrated in figure 1a. The thyristor-controlled reactor consists of a reactor in series with two parallel inverse thyristors. The two inverse parallel thyristors are gated symmetrically. They control the time for which the reactor conducts and thus control the fundamental component of the current. The thyristors conduct on alternate half-cycles of the

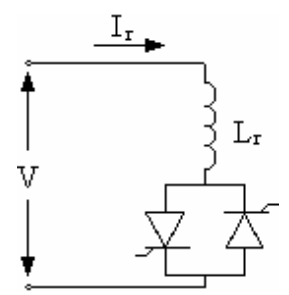

(a)
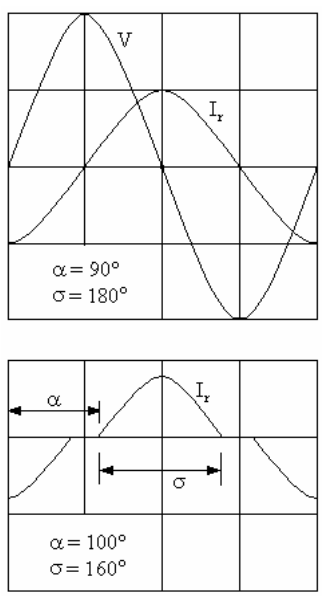

(b)
Figure 1. (a) Main elements of a TCR. (b) Voltage and current waveforms in a TCR. 
supply frequency depending on the firing angle $\alpha$ or conduction angle $\sigma$, which is measured from a zero crossing of voltage. The relation between firing angle and conduction angle as follows:

$$
\sigma=2(\pi-\alpha)
$$

Full conduction is obtained with a firing angle of $90^{\circ}$. Under this condition, the current is reactive and its waveform is purely sinusoidal. There is a partial conduction between $90^{\circ}$ and $180^{\circ}$ as shown in figure $1 \mathrm{~b}$ (Miller 1982). The firing angle is not allowed to have values between $0^{\circ}$ and $90^{\circ}$, causing asymmetrical currents including DC components. When the firing angle $\alpha$ increases from $90^{\circ}$ to $180^{\circ}$, the waveform of the current goes away from the original sinusoidal form. For the condition of balanced loading, TCR produces odd harmonics. In a three-phase system where the TCR's are $\Delta$-connected there are no triplet harmonics injected into the power system (Lasseter \& Lee 1982). However, TCR circuits should not be operated at points near resonance as they would generate conditions causing effective harmonic production (Bohmann \& Lasseter 1986).

\section{Stability and harmonics}

It is necessary to pay attention to energy system stability in the planning, management, and control of electrical power systems for more reliable and "quality" energy (Anderson \& Fouad 1994). Stability in an energy system is defined as the ability of returning to the earlier operating condition after a distortion effect (Begovic \& Phadke 1992). In voltage stability, the amplitude values of the load bus voltages of the system should be kept between determined limit values in both steady-state and transient conditions.

Continuous decrease in the source voltage due to increase in load demand or change in system conditions, causes voltage instability in a system . The main reason for instability is insufficient reactive power not corresponding to the demand. In order to prevent this deficiency, static VAR compensator including TCR should be used (Indulkar et al 1989). Owing to the use of power electronics for static VAR compensation, the power system suffers from instability under some operating conditions (Uzunoglu et al 2000). Due to the nonlinear characteristics of TCR, a new algorithm based on harmonic power flow should be developed to perform steady-state voltage stability analysis.

\section{Harmonic power flow algorithm}

Network voltages and currents can be expressed by Fourier series for the harmonic power flow analysis, which was developed by Xia \& Heydt (1982). Voltages and nonlinear element parameters form the bus variable vector $(\Phi)$, given in

$$
[X]=\left[\left[\dot{V}^{(1)}\right],\left[\dot{V}^{(5)}\right], \ldots,\left[\dot{V}^{(L)}\right],[\phi]\right]^{T} .
$$

In this equation, $L$ is the maximum harmonic order. The mismatch of real and reactive powers for the linear buses (while $k \in\{2, \ldots,(m-1)\}$ ) is defined as,

$$
\left.\begin{array}{l}
\Delta P_{k}=\left(P_{k}\right)_{S P}+F_{r, k}^{(1)} \\
\Delta Q_{k}=\left(Q_{k}\right)_{S P}+F_{i, k}^{(1)}
\end{array}\right\},
$$


where $m$ is the first nonlinear load number and the first bus is the slack bus in this number. $\left(P_{k}\right)_{S P}$ and $\left(Q_{k}\right)_{S P}$ are real and reactive powers at bus $k$ respectively, and $F_{r, k}^{(1)}$ and $F_{i, k}^{(1)}$ are the line fundamental real and reactive powers. The mismatch of real and reactive powers can be calculated for nonlinear buses as,

$$
\left.\begin{array}{l}
\Delta P_{k}^{\text {nonlin }}=\left(P_{k}\right)_{S P}+\sum_{h=1}^{L} F_{r, k}^{(h)} \\
\Delta Q_{k}^{\text {nonlin }}=\left(Q_{k}\right)_{S P}+\sum_{h=1}^{L} F_{i, k}^{(h)}
\end{array}\right\},
$$

where $k \in\{m, m+1, \ldots, n\}$ and $n$ is total number of the buses in the system. $F_{r, k}^{(h)}$ and $F_{i, k}^{(h)}$ can be calculated from (for $h=1,5,7, \ldots, L$ ),

$$
\left.\begin{array}{l}
F_{r, k}^{(h)}=V_{k}^{(h)} \sum_{j=1}^{n} Y_{j k}^{(h)} \cdot V_{j}^{(h)} \cdot \cos \left(\delta_{k}^{(h)}-\theta_{k j}^{(h)}-\delta_{j}^{(h)}\right) \\
F_{i, k}^{(h)}=V_{k}^{(h)} \sum_{j=1}^{n} Y_{j k}^{(h)} \cdot V_{j}^{(h)} \cdot \sin \left(\delta_{k}^{(h)}-\theta_{k j}^{(h)}-\delta_{j}^{(h)}\right)
\end{array}\right\} .
$$

The harmonic phasor voltage the for $k^{\text {th }}$ bus is $\dot{V}_{k}^{(h)}=V_{k}^{(h)} \angle \delta_{k}^{(h)}$ and element $(k, j)$ of the bus admittance matrix calculated for the $h^{\text {th }}$ harmonic frequency is shown in phasor notation as $\dot{Y}_{k j}^{(h)}=Y_{k j}^{(h)} \angle \theta_{k j}^{(h)}$. Here, the mismatch vector for the harmonic power flow is defined as (Grady 1983),

$$
[\Delta \mathbf{M}]=\left[[\Delta W],\left[\Delta I^{(5)}\right],\left[\Delta I^{(7)}\right], \ldots,\left[\Delta I^{(L)}\right],\left[\Delta I^{(1)}\right]\right]^{T},
$$

where $\Delta W$ is the mismatch power vector and $\Delta I^{(h)}$ is the mismatch current vector for the $h^{\text {th }}$ harmonic. The mismatch power is given by

$$
\begin{aligned}
{[\Delta W]=} & {\left[\Delta P_{2}, \Delta Q_{2}, \ldots, \Delta P_{m-1}, \Delta P_{m}^{\text {nonlin }},\right.} \\
& \left.\Delta Q_{m}^{\text {nonlin }}, \ldots, \Delta P_{n}^{\text {nonlin }}, \Delta Q_{n}^{\text {nonlin }}\right] .
\end{aligned}
$$

The mismatch current vector for the fundamental component $(h=1)$ and the harmonic component $(h=5,7, \ldots, L)$, which are the elements of the mismatch vector is given respectively by

$$
\begin{aligned}
{\left[\Delta I^{(1)}\right]=} & {\left[\left(I_{r, m}^{(1)}+g_{r, m}^{(1)}\right),\left(I_{i, m}^{(1)}+g_{i, m}^{(1)}\right),\left(I_{r, m+1}^{(1)}+g_{r, m+1}^{(1)}\right),\right.} \\
& \left.\left(I_{i, m+1}^{(1)}+g_{i, m+1}^{(1)}\right), \ldots,\left(I_{r, n}^{(1)}+g_{r, n}^{(1)}\right),\left(I_{i, n}^{(1)}+g_{i, n}^{(1)}\right)\right]^{r}, \\
{\left[\Delta I^{(h)}\right]=} & {\left[I_{r, 1}^{(h)}, I_{i, 1}^{(h)}, \ldots, I_{r, m-1}^{(h)}, I_{i, m-1}^{(h)},\left(I_{r, m}^{(h)}+g_{r, m}^{(h)}\right),\left(I_{i, m}^{(h)}+g_{i, m}^{(h)}\right),\right.} \\
& \left(I_{r, m+1}^{(h)}+g_{r, m+1}^{(h)}\right),\left(I_{i, m+1}^{(h)}+g_{i, m+1}^{(h)}\right), \ldots, \\
& \left.\left(I_{r, n}^{(h)}+g_{r, n}^{(h)}\right),\left(I_{i, n}^{(h)}+g_{i, n}^{(h)}\right)\right]^{r} .
\end{aligned}
$$

In these equations, $I_{r, k}^{(h)}$ and $I_{i, k}^{(h)}$ are to be zero for the harmonic components at linear buses $(k=1,2, \ldots, m-1)$. 
The Newton-Raphson method is implemented to obtain the correction vector by using the Jakobian matrix. When the mismatch goes to zero for every term of mismatch vector, a solution can be obtained (Arrillaga et al 1983). Thus, by using the Newton-Raphson method, we can get the solution with the desired tolerance (in this study, the tolerance for the mismatch is taken as 0.0001 per unit. The correction vector is given as

$$
[\Delta \mathbf{X}]=[\mathbf{J}]^{-1} \cdot[\Delta \mathbf{M}]
$$

The harmonic power flow analysis algorithm is given in appendix A (Masoum 1991).

\section{Analysis method}

The values obtained from linear power flow analysis are used in steady-state voltage stability analysis performed with conventional methods. It is required to develop a new algorithm to analyse steady-state voltage stability in case the system has a nonlinear element. Synthesis of the harmonic power flow algorithm and the voltage stability algorithm is done, and a new algorithm is developed to realize this analysis (Uzunoglu et al 2002). When the Jakobian of a Newton-Raphson power flow becomes singular, the steadystate voltage stability limit (critical point) of the system can be determined easily and rapidly.

By using data obtained from harmonic power flow, the critical values are calculated for fundamental and harmonic components separately. According to this method, critical transmission angle, $\delta_{\text {critical }}$, critical load voltage, $V_{\text {critical }}$, and critical load power, $P_{\text {critical }}$, are given by (Indulkar 1989)

$$
\delta_{\text {critical }}^{(h)}=1 / 2 \tan ^{-1}\left(K_{1} / K_{2}\right),
$$

where,

$$
\begin{aligned}
K_{1} & =a_{1}^{(h)}\left(b_{2}^{(h)}-b_{1}^{(h)} \tan \varphi^{(h)}\right)+a_{2}^{(h)}\left(b_{1}^{(h)}+b_{2}^{(h)} \tan \varphi^{(h)}\right), \\
K_{2} & =a_{1}^{(h)}\left(b_{1}^{(h)}+b_{2}^{(h)} \tan \varphi^{(h)}\right)+a_{2}^{(h)}\left(-b_{2}^{(h)}+b_{1}^{(h)} \tan \varphi^{(h)}\right), \\
V_{\text {critical }}^{(h)} & =V_{s}^{(h)} / 2 \cdot K_{4},
\end{aligned}
$$

and

$$
\begin{aligned}
& P_{\text {critical }}^{(h)} \\
& =\left\{\left(V_{s}^{(h)}\right)^{2} \cdot\left[2 K_{3} K_{4}-\left(a_{1}^{(h)} b_{1}^{(h)}+a_{2}^{(h)} b_{2}^{(h)}\right)\right]\right\} /\left\{4 \cdot\left(K_{4}\right)^{2} \cdot\left[\left(b_{1}^{(h)}\right)^{2}+\left(b_{2}^{(h)}\right)^{2}\right]\right\},
\end{aligned}
$$

where,

$$
\begin{aligned}
& K_{3}=b_{1}^{(h)} \cos \delta_{\text {critical }}^{(h)}+b_{2}^{(h)} \sin \delta_{\text {critical }}^{(h)}, \\
& K_{4}=a_{1}^{(h)} \cos \delta_{\text {critical }}^{(h)}+a_{2}^{(h)} \sin \delta_{\text {critical }}^{(h)},
\end{aligned}
$$


where $h=1,5,7, \ldots, L$. TCR is modelled suitably for the algorithm developed for the analysis. TCR's current for harmonic components is defined as (Bohmann \& Lasseter 1989)

$$
\begin{aligned}
I_{r}^{(h)}= & \left\{2 \cdot V_{r}^{(h)} / h \cdot X_{r}^{(1)}\right\}\left\{\cos \left(\delta_{r}^{(h)}\right)-\cos \{h \cdot((\pi-\sigma) / 2)\}\right. \\
& \left.+j \cdot\left[\sin \left(\delta_{r}^{(h)}\right)-\sin \{h \cdot((\pi-\sigma) / 2)\}\right]\right\},
\end{aligned}
$$

where $V_{r}$ is reactor terminal voltage, $\delta_{r}$ is its angle, $\sigma$ is thyristor conduction angle, $X_{r}$ is the reactance of the reactor, and $h$ is the harmonic order. Also, reactor admitance depending on thyristor conduction angle can be defined as follows (Bohmann \& Lasseter 1986),

$$
Y_{r}^{(h)}(\sigma)=-j\{\sigma-[\sin (h \sigma)] / h\} /\left\{h X_{r}^{(h)} \pi\right\} .
$$

Equivalent suseptance is calculated by using the capacitor reactance of FC-TCR as,

$$
B^{(h)}(\sigma)=\left[Y_{r}^{(h)}(\sigma)-\left(1 / X_{c}^{(h)}\right)\right] .
$$

In this study, the steady-state voltage stability analysis for power system including FC-TCR was done by the following solution algorithm:

Step 1: The harmonic power flow analysis for the available operation conditions of an example system is done to compute load angle, voltage and power of all buses.

Step 2: Power at all buses except the slack bus and the bus considered for voltage stability are transformed into shunt admittance by using bus voltages obtained from the harmonic load flow for fundamental and harmonic components with the following equations individually,

$$
R=\left(V^{2} / P\right), X=\left(V^{2} / Q\right) \Rightarrow Y=(1 / R)+(1 / j X) .
$$

Step 3: These shunt admittances are added to the bus admittance matrix by taking their directions into consideration (these operations are not performed for the slack bus and the bus considered for voltage stability).

Step 4: After obtaining the new bus admittance matrix, the matrix is reordered. In this case, the elements of the bus considered for voltage stability are in the first row and first column, and the elements of the slack bus are in the second row and second column.

Step 5: The elements at the other buses are reduced to the slack bus and the considered bus by the matrix algebra method. The final reduced matrix $(2 \times 2)$ is obtained by making new orders at the bus admittance matrix as,

$$
\mathbf{Y}_{\text {bus }}=\left[\begin{array}{cc}
\mathbf{K} & \mathbf{L} \\
\mathbf{L}^{\mathbf{T}} & \mathbf{M}
\end{array}\right] \Rightarrow \mathbf{Y}_{\text {bus }}(2 \times 2)=\mathbf{K}-\mathbf{L} \cdot \mathbf{M}^{-1} \cdot \mathbf{L}^{\mathbf{T}} .
$$

Step 6: Critical values are calculated for the final 2-bus system using the Jakobian matrix obtained from singular harmonic power flow $(\operatorname{det}[\mathbf{J}]=0)$. Thus, critical values of the bus examined for the voltage stability are calculated by considering the harmonic components when the FC-TCR is connected to the power system. We also examine how FC-TCR affects the power system voltage stability and the reactive power values. 


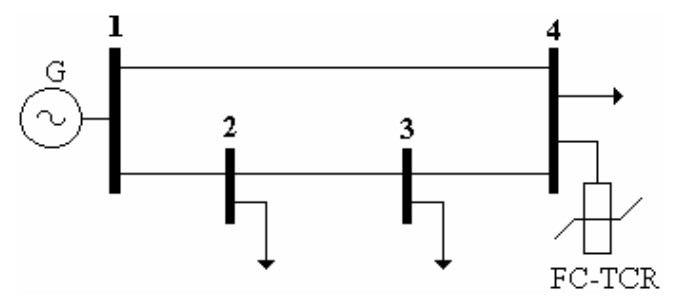

Figure 2. Single line diagram of example system including FC-TCR.

\section{Numerical application}

In this study, the effect of the harmonics on voltage stability has been analysed for a 4-bus power system including FC-TCR (figure 2). Conventional and new voltage stability analyses (depending on harmonic power flow) have been used to perform the study, and the results have been compared.

The per-unit (p.u.) values of lines in the example system have been obtained for $66 \mathrm{kV}$ and 10 MVA base values. The characteristic values of the line and the load data of the systems are given in tables 1 and 2 respectively. All transmission lines are modelled using the same model, the lumped parameters $\pi$ model, and bus- 1 as the slack bus in the example system.

The static compensator with FC-TCR is connected to bus-4. There are no filters at all. The values in the application have been taken as per-unit; Hence, the reactance of TCR $X_{r}=0.55$ p.u., capacitor reactance $X_{c}=2.2$ p.u. and fundamental frequency $f=50 \mathrm{~Hz}$. Analyses have been done and compared using the related algorithms developed based on both linear and harmonic power flows (figures 3-8).

Results of the analyses in which harmonic components are neglected (sinusoidal condition), are denoted by a "+" symbol, while the analyses in which harmonic components are considered (non-sinusoidal condition) are denoted by the "•" in figures $4-8$. When conduction angle, $\sigma$, is between $0^{\circ}$ and $180^{\circ}$, the ratio of harmonic current components $(5,7,11$, 13) / fundamental current component $\left(\mathrm{HDI}_{4}\right.$ ) has been shown (as percentage) in figure 3 for TCR at bus-4. For both conditions, the variation of reactive power for all conduction angles is shown in figure 4.

From the point of steady-state voltage stability, the conduction angles of TCR are $70^{\circ}, 105^{\circ}$ and $160^{\circ}$ and the variations of critical voltage in the frequency domain at bus- 4 are given in figures 5, 6 and 7 respectively.

Variations of critical power values obtained from steady-state voltage stability analysis at bus- 4 and bus- 3 are illustrated in figure 8 and 9 for all conduction angles respectively.

Table 1. Four-bus system impedance and line charging data.

\begin{tabular}{cccc}
\hline From bus & To bus & $Z$, line impedance (p.u.) & $B$, line charging (p.u.) \\
\hline 1 & 2 & $0 \cdot 01+j 0 \cdot 01$ & $j 2 \cdot 1125 e-4$ \\
2 & 3 & $0 \cdot 02+j 0 \cdot 08$ & $j 8 \cdot 4500 e-4$ \\
3 & 4 & $0 \cdot 01+j 0 \cdot 02$ & $j 4 \cdot 2250 e-4$ \\
1 & 4 & $0 \cdot 01+j 0 \cdot 02$ & $j 4 \cdot 2250 e-4$ \\
\hline
\end{tabular}


Table 2. Four-bus system load data.

\begin{tabular}{ccc}
\hline Bus no. & $P_{\text {load }}($ p.u. $)$ & $Q_{\text {load }}($ p.u. $)$ \\
\hline 1 & $0 \cdot 00$ & $0 \cdot 00$ \\
2 & $0 \cdot 10$ & $0 \cdot 10$ \\
3 & $0 \cdot 10$ & $0 \cdot 10$ \\
4 & $0 \cdot 80$ & 0.40
\end{tabular}

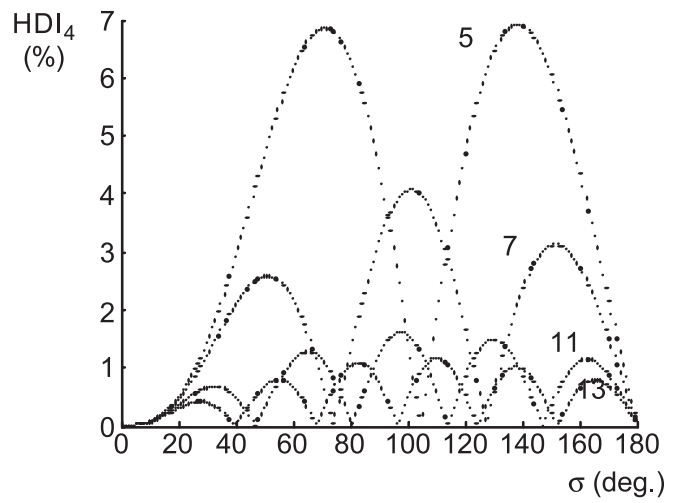

Figure 3. Variations of individual harmonic distortion as a function of conduction angles.

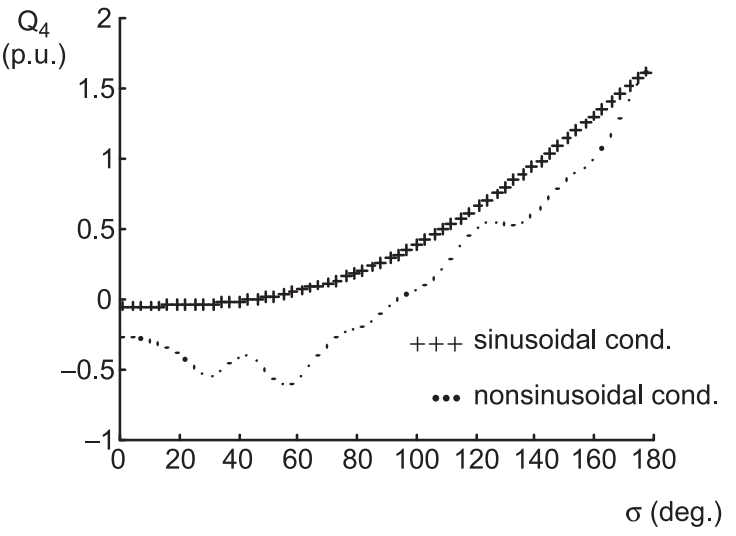

Figure 4. Variations of reactive power at bus-4 for all conduction angles.

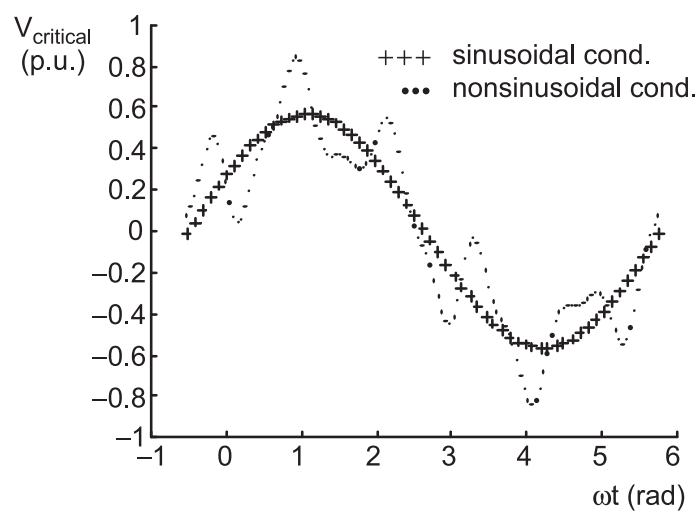

Figure 5. Variations of critical voltage at bus-4 for $\sigma=70^{\circ}$. 

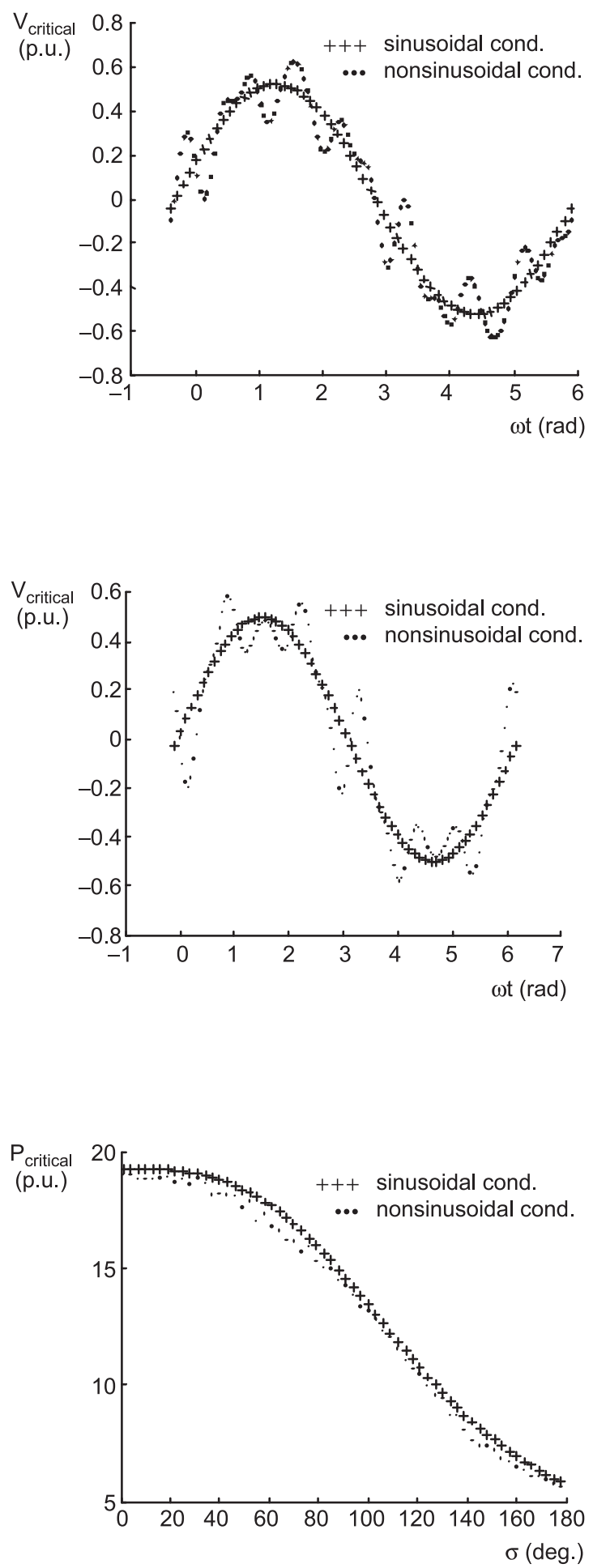

Figure 6. Variation of critical voltage at bus-4 for $\sigma=105^{\circ}$.

Figure 7. Variations of critical voltage at bus-4 for $\sigma=160^{\circ}$.

Figure 8. Critical power values versus conduction angle at bus- 4 . 


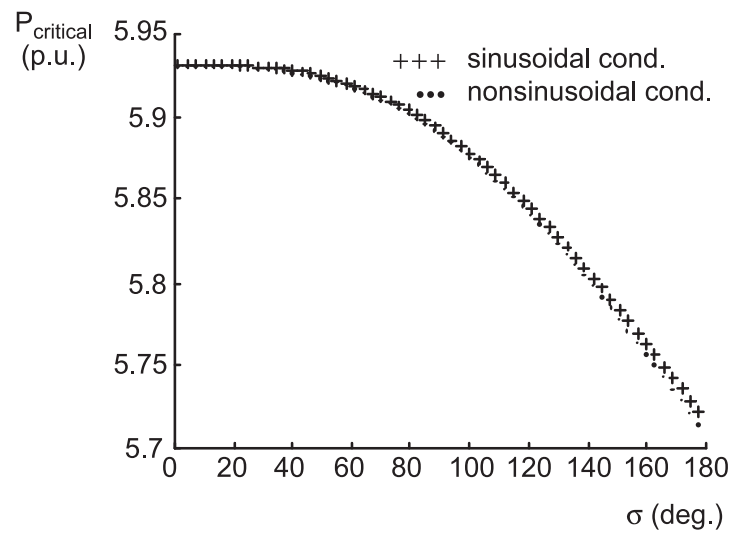

Figure 9. Critical power values versus conduction angle at bus-3.

\section{Conclusions}

We have investigated the effects of harmonics on voltage stability in power systems. The following conclusions are derived from the study.

- Reactive power compensation is very important in terms of voltage stability. Perfect compensation cannot be achieved when the effects of harmonics are not taken into consideration. The simulation results show that there are significant harmonic distortions within the compensator system. Hence, the conduction angle must be changed to obtain the desired reactive power in the system.

- The harmonic distortions in the system are low in properly designed static VAR compensator systems including TCR. This approach of designing for the minimization of harmonic effects helps us in the operation of the system and as a result provides steadystate operations of the system.

- The bus connected with nonlinear elements particularly needs to be studied in terms of stability. The analysis performed in this study indicates that the bus with nonlinear elements is the one most affected by the harmonics.

- When the system connected with static VAR compensator including FC-TCR is examined, it is noticed that some differences occur in critical values obtained from steadystate stability analysis depending on the presence of the harmonic component. This case clearly shows that harmonic components have non-negligible importance in stability.

\section{Appendix A. Harmonic power flow algorithm}

Step 1: Make an initial guess for the fundamental and harmonic bus voltage magnitudes and phase angles $\left(V^{(1)}=1.0\right.$ p.u., $\delta^{(1)}=0$ radian, $V^{(h)}=0.1$ p.u. and $\left.\delta^{(h)}=0\right)$.

Step 2: Compute the nonlinear device currents $g_{r, m}^{(h)}$ and $g_{i, m}^{(h)}$ for nonlinear loads.

Step 3: Evaluate $[\Delta \mathbf{M}](10)-(12)$; if it is a small enough stop.

Step 4: Evaluate $J$ and calculate $[\Delta \mathbf{X}],(13)$ using matrix inversion or forward/backward substitution.

Step 5: Update $[\mathbf{X}],(6),[\mathbf{X}]^{\ell+1}=[\mathbf{X}]^{\ell}-[\Delta \mathbf{X}]^{\ell}(\ell$ is iteration number).

Step 6: Go to step 2. 


\section{References}

Anderson P M, Fouad A A 1994 Power system control and stability (New York: IEEE Press)

Arrillaga J, Arnold C P, Harker B J 1983 Computer modelling of electrical power system (Norwich: John Wiley \& Sons)

Begovic M M, Phadke A G 1992 Control of voltage stability using sensitivity analysis. IEEE Trans. Power Syst. 7: 114-123

Bohmann L J, Lasseter R H 1986 Equivalent circuit for frequency response of a static VAR compensator. IEEE Trans. Power Syst. 1(4): 68-74

Bohmann L J, Lasseter R H 1989 Harmonic interaction in thyristor-controlled reactor circuits. IEEE Trans. Power Delivery 4: 1919-1926

Grady W M 1983 Harmonic power flow studies. Ph D thesis, Purdue University, West Lafayette, IN

Indulkar C S, Viswanathan B, Venkata S S 1989 Maximum power transfer limited by voltage stability in series and shunt compensated schemes for AC transmission systems. IEEE Trans. Power Delivery 2: $1246-1252$

Lasseter R H, Lee S Y 1982 Digital simulation of static VAR system transients. IEEE Trans. Power Apparatus Syst. PAS-101: 4171-4177

Masoum M A S 1991 Generation and propagation of harmonics in power system feeders containing nonlinear loads. Ph D thesis, Department of Electrical and Computer Engineering, University of Colorado, Boulder, CO

Miller T J E 1982 Reactive power control in electric systems (New York: John Wiley \& Sons)

Uzunoglu M, Kocatepe C, Yumurtaci R, Tanrioven M 1999 Harmonics and resonance conditions in thyristor-controlled reactors, ELECO'99, Int. Conf. on Electrical and Electronics Engineering, Bursa, pp. 218-223

Uzunoglu M, Kocatepe C, Yumurtaci R, Gulez K 2000 The various operating conditions, harmonics effects and stability of thyristor-controlled reactor. IEEE TENCON 2000, Kuala Lumpur

Uzunoglu M, Kocatepe C, Yumurtaci R, Gulez K 2002 The effects of harmonics on voltage stability. IEEJ Trans. Inst. Elect. Eng. Jpn. B122: 616-621

Xia D, Heydt G T 1982a Harmonic power flow studies. Part I - Formulation and solution. IEEE Trans. Power Apparatus Syst., PAS-101: 1257-1265

Xia D, Heydt G T 1982b Harmonic power flow studies. Part II - Implementation and practical application. IEEE Trans. Power Apparatus Syst. PAS-101: 1266-1270

Xu W, Marti J R, Dommel H W 1991 Harmonic analysis of systems with static compensators. IEEE Trans. Power Syst. 1(6): 183-190 